\title{
CHARGED CURRENT EVENTS WITH NEUTRAL STRANGE PARTICLES IN HIGH-ENERGY ANTINEUTRINO INTERACTIONS
}

\author{
V.V. AMMOSOV, A.G. DENISOV, P.F. ERMOLOV, G.S. GAPIENKO, V.A. GAPIENKO, \\ V.I. KLUKHIN, V.I. KORESHEV, P.V. PITUKHIN, V.I. SIROTENKO, E.A. SLOBODYUK, \\ Z.V. USUBOV and V.G. ZAETZ
}

Institute of High Energy Physics, Serpukhov, USSR

J.P. BERGE, D. BOGERT, R. ENDORF ${ }^{1}$, R. HANFT, J.A. MALKO, G.I. MOFFATT ${ }^{1}$, F.A. NEZRICK, R. ORAVA and J. WOLFSON

Fermi National Accelerator Laboratory, Batavia, Illinois 60510, USA

\begin{abstract}
V.I. EFREMENKO, A.V. FEDOTOV, P.A. GORICHEV, V.S. KAFTANOV, G.K. KLIGER, V.Z. KOLGANOV, S.P. KRUTCHININ, M.A. KUBANTSEV, I.V. MAKHLJUEVA, V.I. SHEKELJAN and V.G. SHEVCHENKO
\end{abstract}

Institute for Theoretical and Experimental Physics, Moscow, USSR

J. BELL, C.T. COFFIN, B.P. ROE, A.A. SEIDL, D. SINCLAIR and E. WANG

University of Michigan, Ann Arbor, Michigan 48104, USA

Received 23 May 1980

(Revised 5 August 1980)

\begin{abstract}
The results of a study of strange particle production in charged current $\bar{\nu}_{\mu} \mathrm{N}$ interactions in the Fermilab $15 \mathrm{ft}$ bubble chamber filled with a heavy $\mathrm{Ne}-\mathrm{H}_{2}$ mixture are presented. Production rates and average multiplicities of $\mathrm{K}^{0} \mathrm{~s}$ and $\Lambda$ 's as functions of $W^{2}$ and $Q^{2}$ are given. The experimental data agree well with the quark-parton model predictions if a yield of $0.06 \pm 0.02$ of $\mathrm{K}^{0 \text { 's }} \mathrm{s}$ and $A$ 's from charm production is included. Upper limits for D-meson production are given and the shape of the charmed quark fragmentation function is discussed. Inclusive production of the $\mathrm{K}^{*}(890)$ and $\Sigma(1385)$ resonances is measured and it is shown that only about $5 \%$ of the $\mathrm{K}^{\circ}$ mesons and $A$ hyperons results from resonance decays. Relative production rates of neutral strange particles on proton and neutron targets are studied.
\end{abstract}

\section{Introduction}

Strange particles produced by lepton beams can be used as a probe for the production of charmed particles. Since in the GIM charm scheme [1] the charm-tostrange quark transition is favoured we expect to see a reflection of charm production in the events with strange particles in their final states. Non-leptonic decays of charmed particles have already been recorded in $\mathrm{e}^{+} \mathrm{e}^{-}$storage ring experiments [2], photoproduction experiments [3] and $\nu \mathrm{N}$ experiments [4]. Signals for semileptonic charmed particle decays in $\bar{\nu} \mathrm{N}$ interactions have been reported by us in a previous

\footnotetext{
${ }^{1}$ Visitor from University of Cincannati, Cincinnati, Ohio 45221.
} 
study of $\mu$ e events [5]. The quark-parton model (QPM) [6] predicts that essentially all the charmed particle production in $\bar{\nu} \mathrm{N}$ interactions is off sea quarks, and therefore, a study of the scaling variable distributions for inclusive strange particle production can be expected to give indications of charm production [7].

In this paper the results of an investigation of $\mathrm{K}^{0}$ mesons and $A$ hyperons produced in high-energy charged current $(\mathrm{CC})$ antineutrino-nucleon interactions are presented. The inclusive momentum spectra of neutral strange particles observed in this experiment have been published earlier [8]. Here we consider production rates, average multiplicities, the effects of charmed particle production and the production of the resonances $K^{*}(890)$ and $\Sigma(1385)$. Finally, we discuss the relative rates of neutral strange particles produced in interactions with proton and neutron targets.

\section{Experimental details}

The data consist of charged current antineutrino events obtained in the Fermilab $15 \mathrm{ft}$ bubble chamber filled with a $36 \% \mathrm{H}_{2}-64 \% \mathrm{Ne}$ atomic mixture exposed to a wide-band antineutrino beam. A total of 85000 pictures were taken in the doublehorn focused beam with plug and 70000 pictures with a bare target sign selected antineutrino beam. The incident proton energy was $400 \mathrm{GeV}$.

The film was scanned for all neutral-induced interactions with two or more prongs. About 23000 events were fully measured and processed through the geometrical reconstruction program. The scan efficiency was measured in a partial double scan and a multiplicity dependent weight was assigned to very event. The scan efficiency varies from $65 \%$ for the two-prong events up to $\sim 100 \%$ for the events with multiplicity greater than six.

Muons were identified by the external muon identifier supplemented by a selection procedure based on the large transverse momentum of the muon. The overall efficiency for muon identification was $\sim 92 \%$, independent of angle, for muons with momentum greater than $4 \mathrm{GeV} / c$ [9]. The energy of the incident antineutrino was estimated using an average correction for undetected neutral energy loss characteristic of the total sample of events from this experiment ${ }^{\star}$. Finally, the total CC event sample was required to (a) have a positive muon with momentum greater than $4 \mathrm{GeV} / c$, (b) be in a restricted fiducial volume $\left(\sim 17 \mathrm{~m}^{3}\right)$ and (c) have the incident antineutrino energy larger than $10 \mathrm{GeV}$. The number of antineutrino $\mathrm{CC}$ events passing these cuts was about 8200 events.

A subsample of $\mathrm{V}^{0}$ charged current events was defined in the following way. Events having one or more candidates for the decays $\mathrm{K}_{\mathrm{S}}^{0} \rightarrow \pi^{+} \pi^{-}, \Lambda \rightarrow \mathrm{p} \pi^{-}$, or $\bar{\Lambda} \rightarrow \overline{\mathrm{p}} \pi^{+}$associated with the primary vertex were processed through the kinematics

* This method has been described in ref. [9]. Here we use a slightly modified procedure: the parameters $a$ and $b$ in the formula $P_{\mathbf{X}}^{\mathrm{H}}$ (corrected) $=a+b P_{\mathbf{X}}^{\mathrm{H}}$ (visible) were considered as functions of the transverse momentum inbalance in each event, $P_{\mathrm{T}} / P_{\mathrm{X}}$, where $P_{\mathrm{T}}$ and $P_{\mathrm{X}}$ are the transverse and longitudinal total momenta of the event with respect to the incident antineutrino direction. 
TABLE 1

Observed and corrected number of charged current events with neutral strange particles

\begin{tabular}{lccc}
\hline \multicolumn{1}{c}{ Channel } & $\begin{array}{c}\text { Observed } \\
\text { events }\end{array}$ & $\begin{array}{c}\text { Corrected } \\
\text { events }\end{array}$ & $\begin{array}{c}\text { Production } \\
\text { rate }\end{array}$ \\
\hline $\left.\bar{\nu} \mathrm{N} \rightarrow \mu^{+} \mathrm{K}^{0} \mathrm{X}^{\mathrm{a}}\right)$ & 276 & $448 \pm 125$ & $0.055 \pm 0.015$ \\
$\left.\bar{\nu} \mathrm{N} \rightarrow \mu^{+} A \mathrm{X}^{\mathrm{a}}\right)$ & 229 & $301 \pm 42$ & $0.037 \pm 0.005$ \\
$\bar{\nu} \mathrm{N} \rightarrow \mu^{+} \bar{\Lambda} \mathrm{X}$ & 7 & $16 \pm 5$ & $0.002 \pm 0.001$ \\
$\bar{\nu} \mathrm{N} \rightarrow \mu^{+} \mathrm{K}^{0} \overline{\mathrm{K}}^{0} \mathrm{X}$ & 23 & $343 \pm 71$ & $0.042 \pm 0.009$ \\
$\bar{\nu} \mathrm{N} \rightarrow \mu^{+} \Lambda \mathrm{K}^{0} \mathrm{X}$ & 28 & $212 \pm 40$ & $0.026 \pm 0.005$ \\
\hline Total & 563 & $1320 \pm 79$ & $0.162 \pm 0.010$ \\
\hline
\end{tabular}

a) The corrected numbers of events and the production rates for these channels do not include the associated neutral strange particle channels $\mu^{+} K^{0} \overline{\mathrm{K}}^{0} \mathrm{X}$ and $\mu^{+} \Lambda \mathrm{K}^{0} \mathrm{X}$.

fitting program. Details of the $V^{0}$ analysis are given in ref. [8]. Each $V^{0}$ was then weighted to account for the geometrical detection inefficiency, neutral decay modes (including lost $\mathrm{K}_{\mathrm{L}}^{0}$ 's) and scanning and processing inefficiencies. The average weights obtained were 3.9 for $\mathrm{K}_{\mathrm{S}}^{0}, 2.0$ for $\Lambda$ and 2.2 for $\bar{\Lambda}$. Observed raw and corrected numbers of the $\mathrm{V}^{0} \mathrm{CC}$ events in various channels are given in table 1 . Since charged strange particles are not effectively identified, the channels with single $\mathrm{K}^{0}$ or $\Lambda$ may contain contributions from the associated production of neutral and charged strange particles.

\section{Experimental results}

\subsection{PRODUCTION RATES AND AVERAGE MULTIPLICITIES}

The total number of charged current $V^{0}$ events corrected for the experimental inefficiencies, decay modes and potential-length selections is equal to $1320 \pm 79$ events. Normalized to the total $\mathrm{CC}$ event sample this leads to a total $\mathrm{V}^{0}$ event rate of $0.16 \pm 0.01$ for $E_{\bar{\nu}}>10 \mathrm{GeV}$. Event rates for various $\mathrm{V}^{0}$ channels are given in table 1 . For inclusive yields of $\mathrm{K}^{0}$ and $A$ we have:

$$
\begin{aligned}
R_{\mathrm{K}} & =\left(\bar{\nu} \mathrm{N} \rightarrow \mu^{+} \mathrm{K}^{0} \mathrm{X}\right) /\left(\bar{\nu} \mathrm{N} \rightarrow \mu^{+} \mathrm{X}\right)=0.164 \pm 0.009, \\
\boldsymbol{R}_{\Lambda} & =\left(\bar{\nu} \mathrm{N} \rightarrow \mu^{+} \Lambda \mathrm{X}\right) /\left(\bar{\nu} \mathrm{N} \rightarrow \mu^{+} \mathrm{X}\right)=0.063 \pm 0.004, \\
\boldsymbol{R}_{\Lambda / \mathrm{K}} & =\left(\bar{\nu} \mathrm{N} \rightarrow \mu^{+} \Lambda \mathrm{X}\right) /\left(\bar{\nu} \mathrm{N} \rightarrow \mu^{+} \mathrm{K}^{0} \mathrm{X}\right)=0.38 \pm 0.03
\end{aligned}
$$

These rates are compared to the strange particle yields obtained in other neutrino and antineutrino experiments in table 2.

Since the charged K's are not identified it is not possible to determine the single strange particle production rate in our data. However, we may estimate the number 
TABLE 2

Comparison of the inclusive strange particle production rates in different $\nu$ and $\bar{\nu}$ experiments

\begin{tabular}{lccc}
\hline Ref. & $R_{\mathrm{K}}=N_{\mathbf{K}^{0}} / N_{\mathrm{CC}}$ & $R_{\Lambda}=N_{\Lambda} / N_{\mathrm{CC}}$ & $R_{\Lambda / \mathbf{K}}=N_{\Lambda} / N_{\mathbf{K}^{0}}$ \\
\hline This exp. & $0.16 \pm 0.01$ & $0.06 \pm 0.01$ & $0.38 \pm 0.03$ \\
$\nu \mathrm{N}[10]$ & $0.14 \pm 0.02$ & $0.05 \pm 0.01$ & $0.37 \pm 0.06$ \\
$\nu \mathrm{p}[11]$ & $0.14 \pm 0.02$ & $0.06 \pm 0.02$ & $0.40 \pm 0.11$ \\
$\bar{\nu} \mathbf{p}[12]$ & $0.15 \pm 0.04$ & $0.06 \pm 0.02$ & $0.40 \pm 0.16$ \\
$\nu \mathrm{p}[14]$ & $0.15 \pm 0.02$ & $0.04 \pm 0.01$ & $0.30 \pm 0.07$ \\
\hline
\end{tabular}

of $\Delta S=\Delta Q$ events which contain a $\Lambda$ by assuming equal rates for the $\Lambda \mathrm{K}^{0}$ and $A \mathrm{~K}^{+}$ (associated) production. With this assumption we obtain the $\Lambda$ production rate in the $\Delta S=\Delta Q$ processes of $(1.1 \pm 0.7) \%$ of the total CC sample. This value is compatible with the QPM predictions of $\sim 4 \%$ for the total single strange particle production in $\bar{\nu} \mathrm{N}$ interactions calculated with our experimental conditions and the quark densities of ref. [16].

In principle, the average strange particle multiplicities may be functions of both $W^{2}$ and $Q^{2}$, where $W$ is the total hadronic invariant mass and $Q^{2}$ is the square of the four-momentum transfer between the incident antineutrino and the outgoing muon. The $W^{2}$ dependence of the $\mathrm{K}^{0}$ and $\Lambda$ multiplicity is shown in figs. $1 \mathrm{a}, \mathrm{b}$. For comparison, data from neutrino-nucleon [10] charged current interactions are also plotted in these figures. A strong rise with $W^{2}$ is seen for both $\mathrm{K}^{0}$ and $\Lambda$ multiplicities

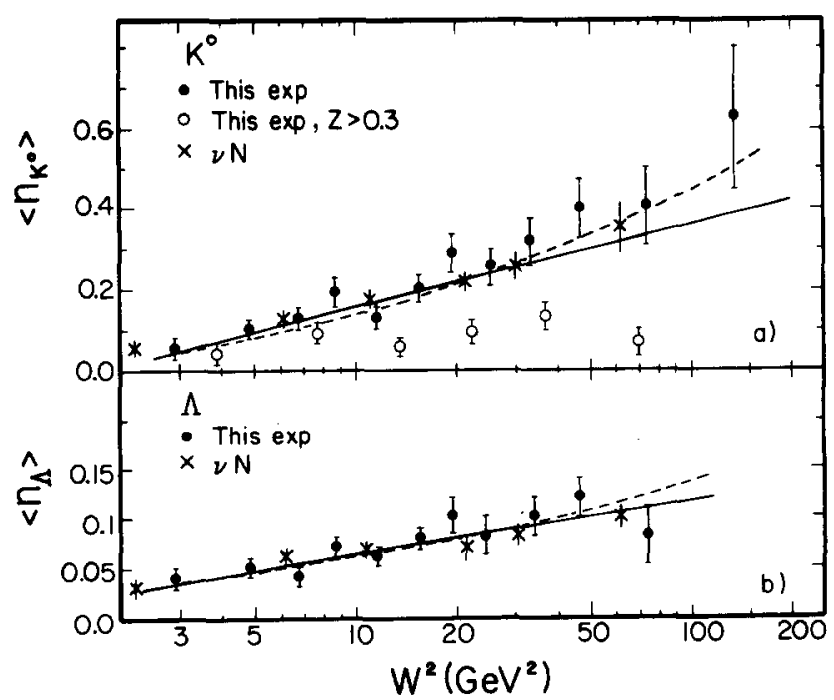

Fig. 1. Average multiplicity of (a) $\mathrm{K}^{0}$, s and (b) $A$ 's as a function of $W^{2}$. Solid and dashed lines represent the results of the logarithmic and power-law fits, respectively, described in the text. The neutrino-nucleon data are also shown. The open data points give $\mathrm{K}^{0}$ multiplicity in the region $z>0.3$. 
and a logarithmic parametrization of the data over the range $2<W^{2}<200 \mathrm{GeV}^{2}$ for $\mathrm{K}^{0}$ 's and $2<W^{2}<100 \mathrm{GeV}^{2}$ for $\Lambda$ 's yields

$$
\begin{aligned}
& \left\langle n_{\mathrm{K}}\right\rangle=(-0.068 \pm 0.017)+(0.094 \pm 0.009) \ln W^{2}, \\
& \left\langle n_{\Lambda}\right\rangle=(0.008 \pm 0.012)+(0.024 \pm 0.005) \ln W^{2},
\end{aligned}
$$

with $\chi^{2} / \mathrm{ND}=17.4 / 10$ for $\mathrm{K}^{0}$ and $\chi^{2} / \mathrm{ND}=7.5 / 9$ for $\Lambda$ data. A fit to the expression $\langle n\rangle=a+b W^{1 / 2}$ (ref. [15]) gives the following results:

$$
\begin{aligned}
& \left\langle n_{\mathrm{K}}\right\rangle=(-0.250 \pm 0.033)+(0.220 \pm 0.021) W^{1 / 2}, \\
& \left\langle n_{A}\right\rangle=(-0.029 \pm 0.019)+(0.052 \pm 0.011) W^{1 / 2},
\end{aligned}
$$

with $\chi^{2} / \mathrm{ND}=13.0 / 10$ for $\mathrm{K}^{0}$ and $\chi^{2} / \mathrm{ND}=7.7 / 9$ for $\Lambda$ data, respectively. It is apparent that the behaviour of $\left\langle n_{\mathrm{K}}\right\rangle$ and $\left\langle n_{A}\right\rangle$ is similar for both $\bar{\nu} \mathrm{N}$ and $\nu \mathrm{N}$ data.

In fig. 1a we also present the $W^{2}$ dependence of $\left\langle n_{\mathrm{K}}\right\rangle$ for $\mathrm{K}^{0}$ mesons with $z>0.3$, where $z$ is defined for an individual hadron $\mathrm{h}$ by $z=E_{\mathrm{h}} / \nu$ where $E_{\mathrm{h}}$ is the energy of hadron $h$, and $\nu$ is the total hadronic energy in the laboratory system. This selection is applied to remove most $\mathrm{K}^{0}$ s associated with the target fragmentation region. We can see that there is no clear dependence of $\left\langle n_{\mathrm{K}}\right\rangle$ on $W^{2}$. This is in agreement with the $\nu \mathrm{p}$ data in the current fragmentation region [13].

In fig. 2 we show the neutral strange particle multiplicities as a function of $Q^{2}$. The $\mathrm{K}^{0}$ and $\Lambda$ data suggest a slow increase in the mean multiplicity with increasing $Q^{2}$. The increase might be related to the strong $W^{2}$ dependence of the $V^{0}$ multiplicities as observed above. To check this we present the values of $\left\langle n_{\mathrm{K}}\right\rangle$ and $\left\langle n_{A}\right\rangle$ as a function of

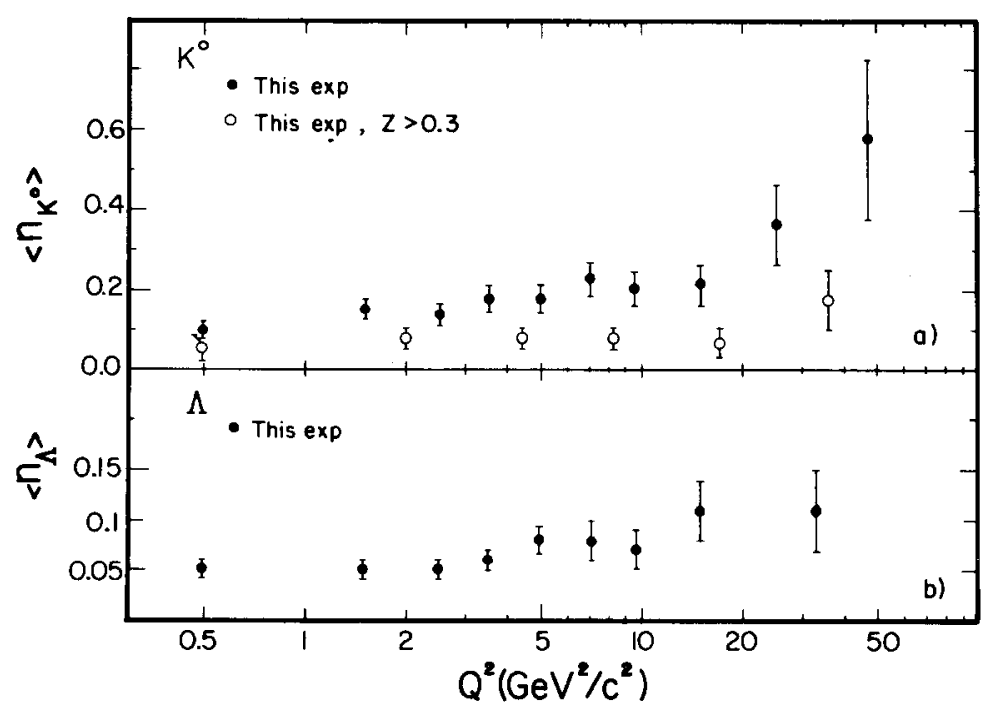

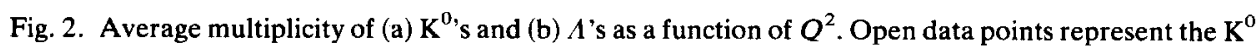
data in the current fragmentation region $z>0.3$. 


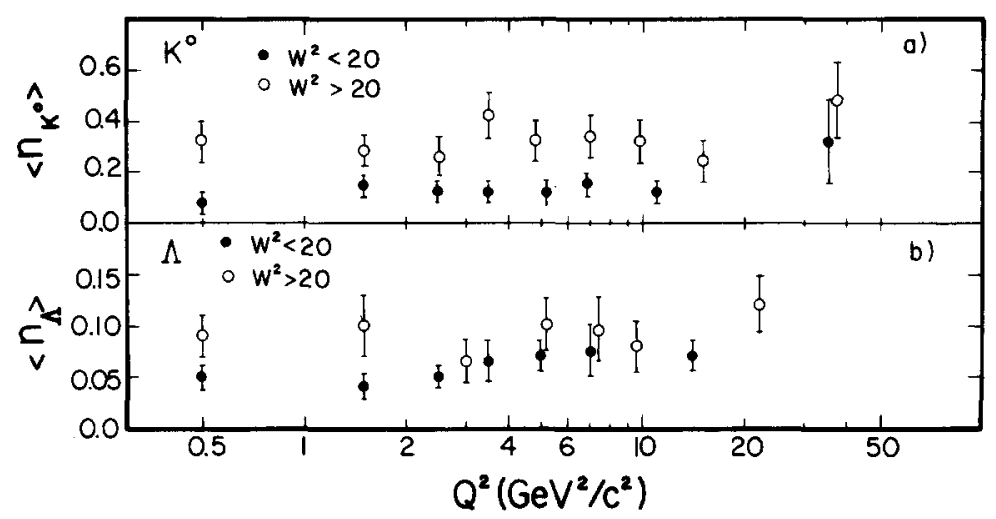

Fig. 3. $Q^{2}$ dependence of the mean multiplicity of the (a) $\mathrm{K}^{0 \text { 's }}$ and (b) $A$ 's. The black data points show the multiplicities in the low- $W^{2}$ region $\left(<20 \mathrm{GeV}^{2}\right)$, the open data points give the data in the high- $W^{2}$ region $\left(>20 \mathrm{GeV}^{2}\right)$.

$Q^{2}$ in figs $3 \mathrm{a}, \mathrm{b}$ for two $W^{2}$ regions: $W^{2}<20 \mathrm{GeV}^{2}$ and $W^{2}>20 \mathrm{GeV}^{2}$. With these $W^{2}$ selections no significant dependence of $\left\langle n_{\mathrm{V}}\right\rangle$ on $Q^{2}$ is observed.

The behaviour of the mean $\mathrm{K}^{0}$ multiplicity in the current fragmentation region $(z>0.3)$ is shown in fig. $2 \mathrm{a}$ by open data points. Within the limits of statistics, our experimental data are consistent with no dependence on $Q^{2}$ in agreement with $\nu \mathrm{p}$ data [13]. The absence of a $Q^{2}$ dependence is expected in the simplest version of the quark-parton picture of quark fragmentation.

\subsection{INDIRECT SEARCH FOR CHARM EFFECTS}

In the QPM the main part of the antineutrino-nucleon cross section comes from valence quark scattering and the cross section therefore has the $x$-distribution characteristic of the valence quarks and a $y$-dependence, $\mathrm{d} \sigma / \mathrm{d} y \sim(1-y)^{2}$. On the other hand, charm production occurs only off sea antiquarks and should be concentrated at low $x$ and have a flat $y$-distribution. Here $x$ and $y$ are the usual Bjorken scaling variables $\left(x=Q^{2} / 2 M \nu, y=\nu / E_{\bar{v}}\right)$. Since charmed particles decay primarily into strange particles, they can be used as charm triggers. Specifically, one can look for an excess of events with $\mathrm{K}^{0 \text {, }}$ and $\Lambda$ 's at low $x$ and large $y$. The inclusive strange particle spectra integrated over $x$ and $y$, on the other hand, are less sensitive to the effects of charm production.

The antineutrino charged current differential scattering cross section off an isoscalar target can be written in the QPM as

$$
\sigma_{\text {tot }}(x, y) \sim(u+d)(1-y)^{2}+(\bar{u}+\bar{d}+2 \bar{s})
$$

where $u, d, \bar{u}, \ldots$ are the quark densities $x u(x), x d(x), x \bar{u}(x), \ldots$ for the corresponding quarks in a proton. We use the parametrization of Field and Feynman [16] and 
neglect the contribution of charmed quarks in the sea. We can identify the various contributions to the total $\mathrm{V}^{0}\left(\mathrm{~K}^{0}\right.$ and $\Lambda$ ) production as follows:

(a) Non-charm single $\mathrm{V}^{0}$ production, $\sigma_{1}$ :

$$
\sigma_{1}(x, y) \sim\left\{(u+d)(1-y)^{2}+2 \bar{s}\right\} \sin ^{2} \theta_{\mathrm{C}},
$$

where the first term comes from the $u \rightarrow s$ transition and the second term from the $\overline{\mathbf{s}} \rightarrow \overline{\mathbf{u}}$ transition. The $\mathrm{V}^{0}$ results from the left-over s quark in the $s \bar{s}$ pair.

(b) Charm-induced $\mathrm{V}^{0}$ production, $\sigma_{2}$ :

$$
\sigma_{2}(x, y) \sim(\bar{u}+\bar{d}) \sin ^{2} \theta_{\mathrm{C}}+4 \bar{s} \cos ^{2} \theta_{\mathrm{C}},
$$

where the first term comes from the $\bar{d} \rightarrow \bar{c}$ transition and the second from the $\bar{s} \rightarrow \bar{c}$ transition. The second term gives rise to two strange particles: one from the charmed particle decay and another from the left-over s quark in the sea.

(c) Finally, we must include the associated strange particle production that results from the fragmentation of the non-strange quarks:

$$
\sigma_{3}(x, y) \sim(u+d)(1-y)^{2} \cos ^{2} \theta_{\mathrm{C}}+(\bar{u}+\bar{d}) \cos ^{2} \theta_{\mathrm{C}} .
$$

This term also gives rise to two strange particles in the final state. We assume that the probability of associated production depends on $W^{2}$ as $A+B \ln W^{2}$ as noted by Messing ${ }^{\star}$. The final results are insensitive to the exact choice of $A$ and $B$.

These fundamental transitions $\sigma_{1}, \sigma_{2}, \sigma_{3}$ must be multiplied by the strange quark multiplicity and the $\mathrm{K}^{0}$ and $\Lambda$ detection efficiencies in order to predict the absolute $x$ and $y$ distributions of the neutral strange particles. In the present analysis we assume that the detection efficiencies do not depend on the quark transitions.

To compare with our data, the resulting cross sections must be integrated over the antineutrino flux, modified according to the experimental $x$ and $y$ selection efficiencies and finally, $\sigma_{1}, \sigma_{2}$, and $\sigma_{3}$ must be multiplied by an assumed threshold behaviour. Therefore, we introduce a set of functions of the scaling variable $x$ :

$$
\begin{aligned}
f_{i}(x) & \equiv \iint \sigma_{i}(x, y) E_{\bar{\nu}} \phi\left(E_{\bar{\nu}}\right) \theta_{\mathrm{s}} \theta_{i} \mathrm{~d} E_{\bar{\nu}} \mathrm{d} y, \\
f_{\mathrm{tot}}(x) & \equiv \iint \sigma_{\mathrm{tot}}(x, y) E_{\bar{\nu}} \phi\left(E_{\bar{\nu}}\right) \theta_{\mathrm{s}} \mathrm{d} E_{\bar{\nu}} \mathrm{d} y, \\
F_{i}(x) & =f_{i}(x) / f_{\mathrm{tot}}(x), \\
\int f_{i}(x) \mathrm{d} x & =1, \quad \int f_{\mathrm{tot}}(x) \mathrm{d} x=1, \quad i=1,2,3 .
\end{aligned}
$$

A similar set of the functions for the variable $y$ may be introduced by the replacement $x \leftrightarrow y$. Here $\phi\left(E_{\bar{v}}\right)$ is the relative antineutrino flux obtained from our CC data, $\theta_{\mathrm{s}}$ is

* We have used the following values for the parameters $A$ and $B: A=-0.005, B=0.0175$ given in ref. [17]. 
the step-function $\theta_{\mathrm{s}}=\theta\left(E_{\bar{\nu}}-E_{\bar{\nu}}^{\mathrm{min}}\right) \theta\left(p_{\mu}-p_{\mu}^{\mathrm{min}}\right)$ which takes into account our experimental selections $E_{\bar{\nu}}^{\min }=10 \mathrm{GeV}$ and $P_{\mu}^{\min }=4 \mathrm{GeV} / c$. The other stepfunctions $\theta_{i}=\theta\left(W-W_{i}^{\text {th }}\right)$ take into account the threshold effects of the single strange particle production, charmed production and associated strange particle production, respectively. For charm production a sharp threshold behaviour (so called "fast rescaling") was assumed with a threshold value of $W^{\text {th }}=2.5 \mathrm{GeV} / c^{2}$.

We can now write the $\mathrm{V}^{0}$ multiplicity as a function of $x$ (or $y$ ) in the following form:

$$
\frac{\mathrm{d} N\left(\mathrm{~V}^{0}\right) / \mathrm{d} x}{\mathrm{~d} N(\mathrm{CC}) / \mathrm{d} x}=\left\langle n_{1}^{\mathrm{v}}\right\rangle F_{1}(x)+\left\langle n_{2}^{\mathrm{v}}\right\rangle F_{2}(x)+\left\langle n_{3}^{\mathrm{v}}\right\rangle F_{3}(x),
$$

where $N\left(\mathrm{~V}^{0}\right)$ and $N(\mathrm{CC})$ are the numbers of $\mathrm{V}^{0}$ s and charged current events, respectively, and $\left\langle n_{i}^{\mathrm{V}}\right\rangle$ is the partial average $\mathrm{V}^{0}$ multiplicity from the production source $i$, integrated over the entire $x$ and $y$ regions. We have used the above expressions to make a simultaneous fit to our experimental $x$ - and $y$-distributions. The results are shown in figs. $4 \mathrm{a}$, b, where the solid lines represent the results of the complete fit and the dashed lines show the non-charm part of the fit. Because the shapes of functions $F_{1}$ and $F_{3}$ are very similar in the central regions of $x$ and $y$, the average $\mathrm{V}^{0}$ multiplicity from the single production $\left\langle n_{1}^{\vee}\right\rangle$, was fixed in the fit. The value of $\left\langle n_{1}^{\mathrm{V}}\right\rangle$ was obtained with an assumption that the charged and neutral kaons are produced with an equal probability and that the number of $\Lambda$ 's and $\Sigma^{0}$ 's is
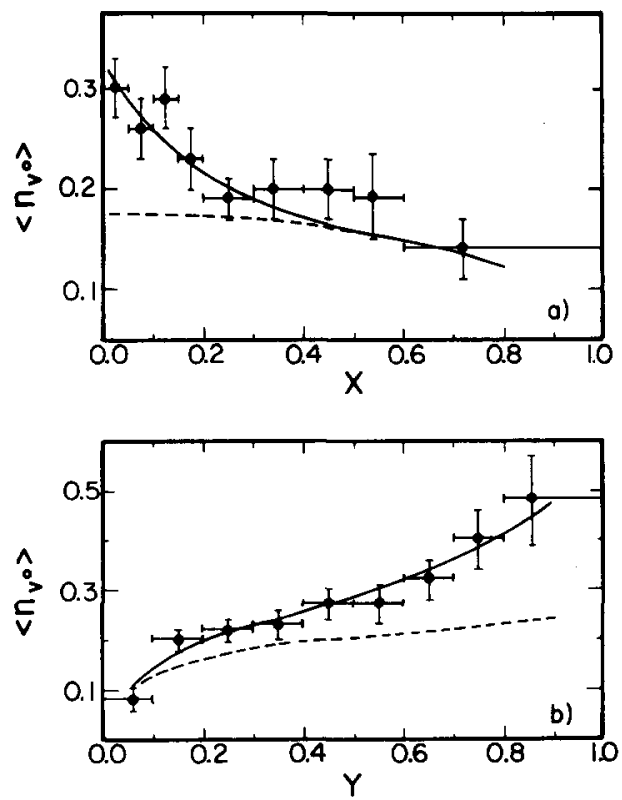

Fig. 4. Mean multiplicity of $\mathrm{V}^{0} \mathrm{~s}\left(\mathrm{~K}^{0 \text { 's }} \mathrm{s}\right.$ and $\Lambda$ 's) as a function of (a) $x$ and (b) $y$. Solid curves result from the fit with the charm term (see the text). Dashed lines show the non-charm part of this fit. 
approximately equal to the number of $\Sigma^{ \pm}$s. The QPM then gives, with these assumptions, $\left\langle n_{1}^{\mathrm{V}}\right\rangle \simeq 0.021$.

The fit with the charm term gives a value of $\chi^{2} / \mathrm{ND}=21.0 / 17$ which corresponds to a probability of $P\left(\chi^{2}\right) \simeq 23 \%$. This result should be compared with $P\left(\chi^{2}\right) \sim 10^{-4}$ which was obtained by fitting the data to the same form without the charm term. Thus we see that the observed behaviour of the average $\mathrm{V}^{0}$ multiplicity as a function of $x$ and $y$ can be described by the QPM where the charmed quarks are produced off sea quarks and decay into strange particles according to the GIM mechanism.

The fit predicts that the total charm contribution to the $\mathrm{K}^{0}$ and $\Lambda$ multiplicity is

$$
\left\langle n_{2}^{\mathrm{v}}\right\rangle=0.06 \pm 0.02 \text {. }
$$

If we assume that the $\mathrm{K}^{0}$ and $\Lambda$ rates measured represent one half of the charm production, then the total charm contribution to the $\bar{\nu} \mathrm{N}$ cross section is $(6 \pm 2) \%$. This rate is compatible with the observed $\mu^{+} \mathrm{e}^{-}$rate in this experiment of $(0.36 \pm$ $0.11) \%[5]$ and the charmed meson semileptonic branching ratios.

\subsection{CHARM FRAGMENTATION}

Models describing hadron production in the deep-inelastic reactions are based on the idea of quark fragmentation [16]. To obtain predictions for the strange particle production in these models one has to know the shape of the charmed quark fragmentation function. Here we use our data on inclusive $\mathrm{K}^{0}$ production in the high $z$ region $(z>0.3)$ and compare them with the QPM predictions.

The inclusive $\mathrm{K}^{0}$ cross sections for the current fragmentation region $(z>0.3)$ is given in the QPM as

$$
\begin{aligned}
\frac{\mathrm{d}^{3} \sigma}{\mathrm{d} x \mathrm{~d} y \mathrm{~d} z}= & \frac{G^{2} M E}{\pi}\left\{(u+d)(1-y)^{2}\left(D_{\mathrm{d}}^{\mathrm{K}}(z) \cos ^{2} \theta_{\mathrm{C}}+D_{\mathrm{s}}^{\mathrm{K}}(z) \sin ^{2} \theta_{\mathrm{C}}\right)\right. \\
& +(\bar{u}+\bar{d})\left(D_{\overline{\mathrm{u}}}^{\mathrm{K}}(z) \cos ^{2} \theta_{\mathrm{C}}+D_{\overline{\mathrm{c}}}^{\mathrm{K}}(z) \sin ^{2} \theta_{\mathrm{C}}\right) \\
& \left.+2 \bar{s}\left(D_{\overline{\mathrm{c}}}^{\mathrm{K}}(z) \cos ^{2} \theta_{\mathrm{C}}+D_{\overline{\mathrm{u}}}^{\mathrm{K}}(z) \sin ^{2} \theta_{\mathrm{C}}\right)\right\}
\end{aligned}
$$

where $u, d, \ldots, \bar{s}$ are quark densities (see subsect. 3.2), $D_{\mathrm{q}}^{\mathrm{K}}(z)$ is the fragmentation function of a quark $q$ into the $\mathrm{K}^{0}$ meson and $\theta_{\mathrm{C}}$ is the Cabibbo angle. Our data do not allow us to determine the four fragmentation functions in the above formula. However, in the region $x>0.3$ the antiquark densities are negligible. Normalizing to the total charged current sample, we have for the region $x>0.3$

$$
\frac{1}{N_{\mathrm{CC}}} \frac{\mathrm{d} n}{\mathrm{~d} z}=D_{\mathrm{d}}^{\mathrm{K}}(z) \cos ^{2} \theta_{\mathrm{C}}+D_{\mathrm{s}}^{\mathrm{K}}(z) \sin ^{2} \theta_{\mathrm{C}},
$$

where $N_{\mathrm{CC}}$ is the number of charged current events and $n$ is the number of $\mathrm{K}^{0}$ mesons. 
Using our data in the region $x>0.3, z>0.3$ we obtain

$$
\int_{0.3}^{1}\left(D_{\mathrm{d}}^{\mathrm{K}}(z) \cos ^{2} \theta_{\mathrm{C}}+D_{\mathrm{s}}^{\mathrm{K}}(z) \sin ^{2} \theta_{\mathrm{C}}\right) \mathrm{d} z=0.068 \pm 0.011 .
$$

This number agrees well with the value of $\sim 0.075$ deduced from the measurements of $D_{\mathrm{d}}^{\mathrm{K}}(z)$ and $D_{\mathrm{s}}^{\mathrm{K}}(z)$ in a recent ep experiment [18].

Next we use our inclusive $\mathbf{K}^{0}$ data to search for the effects of charmed particle production. The $z$-distribution of the $\mathrm{K}^{0}$, $s$ obtained by integrating the differential cross section over the entire $x$ and $y$ region is not sensitive to the charm term. This insensitivity comes from the small contribution of sea antiquarks to the total cross section. Therefore, we have studied the inclusive $\mathrm{K}^{0}$ yield as a function of $x$ and $y$ in the current fragmentation region $(z>0.3)$.

For the term $D_{\mathrm{d}}^{\mathrm{K}} \cos ^{2} \theta_{\mathrm{C}}+D_{\mathrm{s}}^{\mathrm{K}} \sin ^{2} \theta_{\mathrm{C}}$ we use the result obtained above from our data and for the $D_{\overline{\mathrm{u}}}^{\mathrm{K}}(z)$ fragmentation function we take the function determined in an ep experiment [18]. The only unknown quantity is then the charmed quark fragmentation function $D_{\overline{\mathbf{c}}}^{\mathrm{K}}(z)$. This function should describe a two-step process where the charmed quark first dresses itself into a charmed particle. The charmed particle then decays into the observed final-state hadrons. For our calculations we have assumed that these intermediate charmed particles are all $\mathrm{D}(1860)$ mesons, $\mathrm{D}^{-}$or $\overline{\mathrm{D}}^{0}$, and that the observed $\mathrm{K}^{0}$ mesons come from the subsequent $\mathrm{D}(1860)$ decays.
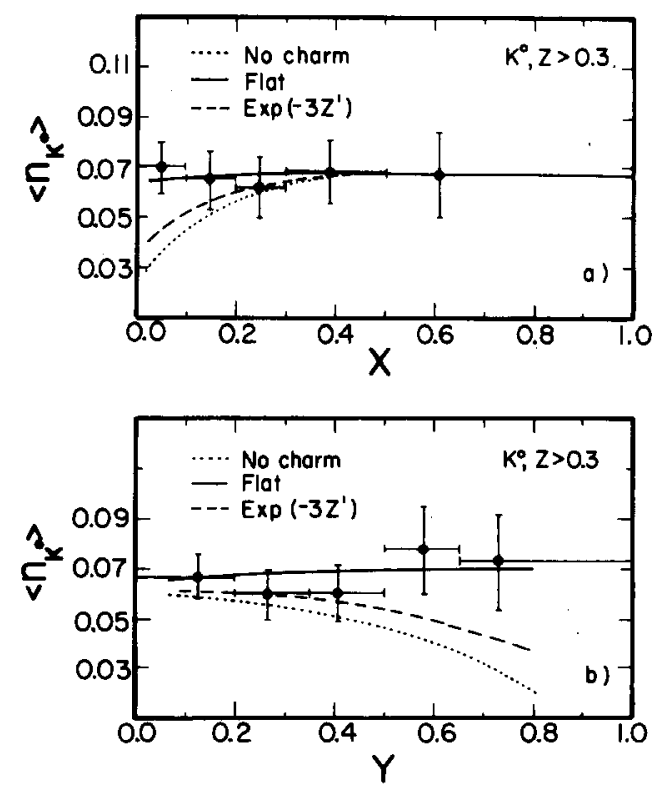

Fig. 5. The (a) $x$ and (b) $y$ dependences of the $\mathrm{K}^{0}$ mean multiplicity in the current fragmentation region $z>0.3$. Dotted lines represent the QPM predictions without charm, dashed lines give the predictions with an exponential charmed quark fragmentation function and solid lines with a flat charmed quark fragmentation function (see the text). 
Calculation of the charmed quark fragmentation function, $D_{\overline{\mathbf{c}}}^{\mathbf{K}}(z)$ depends on the fragmentation function of a charmed quark into the $\mathrm{D}(1860)$ mesons, $D_{\mathfrak{c}}^{\mathrm{D}}\left(z^{\prime}\right)$, where $z^{\prime}$ is the fractional hadronic energy of the charmed meson, and the decay kinematics for the various $\mathrm{D}(1860) \rightarrow \mathrm{K}^{0}+\mathrm{X}$ final states. For $D_{\mathrm{c}}^{\mathrm{D}}\left(z^{\prime}\right)$ we have tried both a constant form and an exponential, $\mathrm{e}^{-3 z^{\prime}}$, which were suggested by Lai [19] to provide a reasonable description of previous dilepton data. For the $D(1860) \rightarrow K^{0}+X$ branching ratios the existing experimental data [20] supplemented by theoretical predictions [21] have been used. Finally, we have used phase space calculations for D meson decays into $\mathrm{K}^{\mathbf{0}}$ 's.

Results of the above model, modified to meet our experimental conditions and the antineutrino energy spectrum, are shown in figs. $5 \mathrm{a}$, b where we present the mean $\mathrm{K}^{0}$ multiplicity in the current fragmentation region as a function of $x$ and $y$, respectively. The model predictions are shown with a flat charmed quark fragmentation function (solid curve), with an exponential fragmentation function (dashed line) and with the assumption of no charm production (dotted curve). The data have a strong predictive strength in the small $x$ and high $y$ regions and they are clearly inconsistent with the no-charm hypothesis. Because the statistics are limited we can not exclude either of the two assumed charmed quark fragmentation functions, but there is an indication that the flat function describes the experimental data better.

\subsection{INCLUSIVE MASS SPECTRA}

Using our results above, we should expect a rate of $\sim 6 \%$ for charmed particle production in $\bar{\nu} \mathrm{N}$ interactions. Therefore, it might be possible to see signals from charmed particle production in the effective mass distributions. The large number of competing charmed particle decay modes will dilute the signal in any separate channel and the particle combinations in the high multiplicity events are an additional problem. We have searched for evidence of $D^{-}$and $\overline{\mathbf{D}}^{0}$ production in the inclusive $\mathrm{K}^{0}$ sample by studying the relevant invariant mass distributions.

The $\mathrm{K}^{0} \pi^{-}$invariant-mass spectrum is shown in fig. 6a. No signal is apparent near the $\mathrm{D}^{-}(1868)$ mass in this distribution. A background estimation by a polynomial fit resulted in the following upper limit for the $\mathrm{D}^{-}(1868)$ production in CC interactions:

$$
\frac{\bar{\nu} \mathrm{N} \rightarrow \mu^{+} \mathrm{D}^{-} \mathrm{X}, \mathrm{D}^{-} \rightarrow \mathrm{K}^{0} \pi^{-}}{\bar{\nu} \mathrm{N} \rightarrow \mu^{+} \mathrm{X}}<0.2 \%, \quad(90 \% \text { c.l. upper limit }) .
$$

The $\mathrm{K}^{0} \pi^{+} \pi^{-}$effective mass distribution is presented in fig. 7. There is no apparent signal from the $\overline{\mathrm{D}}^{0}(1863)$ decays. Using the same procedure for background estimation as before we obtain the following upper limit for $\overline{\mathrm{D}}^{\circ}(1863)$ production:

$$
\frac{\bar{\nu} \mathrm{N} \rightarrow \mu^{+} \overline{\mathrm{D}}^{0} \mathrm{X}, \overline{\mathrm{D}}^{0} \rightarrow \mathrm{K}^{0} \pi^{+} \pi^{-}}{\bar{\nu} \mathrm{N} \rightarrow \mu^{+} \mathrm{X}}<0.3 \%, \quad(90 \% \text { c.l. upper limit }) .
$$



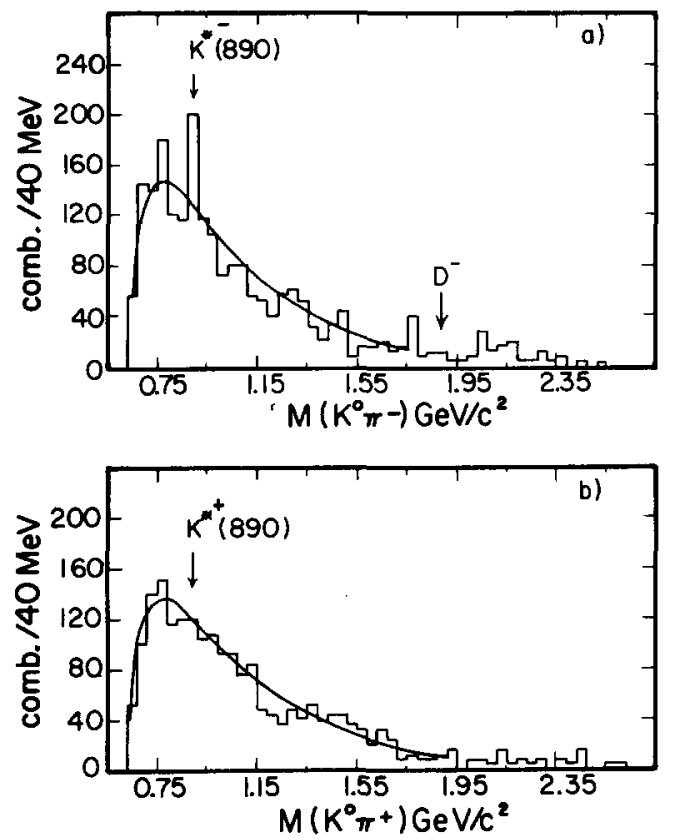

Fig. 6. The invariant-mass distribution for (a) $\mathrm{K}^{0} \pi^{-}$, (b) $\mathrm{K}^{0} \pi^{+}$. An estimate of the background is shown by the solid curve. In figs. 6,7 and 8 weighted combinations are presented.

Both these results may be compared with the $\mathrm{D}^{+}$and $\mathrm{D}^{0}$ rates obtained in $\nu \mathrm{N}$ interactions: $(0.1 \pm 0.1) \%$ for $\mathrm{D}^{+}$production [4] and $(0.40 \pm 0.15) \%$ for $\mathrm{D}^{0}$ production [22], respectively. By using the previously measured $\mathrm{D}^{-} \rightarrow \mathrm{K}^{0} \pi^{-}$and $\overline{\mathrm{D}}^{0} \rightarrow$ $\mathrm{K}^{0} \pi^{+} \pi^{-}$branching ratios [20] of $\sim 1.5 \%$ for $\mathrm{D}^{-}$and $\sim 4.4 \%$ for $\overline{\mathrm{D}}^{0}$ we obtain the upper limits of $\sim 13 \%$ for total $\mathrm{D}^{-}$production and $\sim 7 \%$ for total $\overline{\mathrm{D}}^{0}$ production in antineutrino-nucleon charged current interactions.

The $\mathrm{K}^{0} \pi^{-}$and $\mathrm{K}^{0} \pi^{+}$invariant mass distributions are presented in figs. $6 \mathrm{a}, \mathrm{b}$, respectively. A signal from the $\mathrm{K}^{*-}(890)$ production is seen (fig. 6a), but there is no evidence for $\mathrm{K}^{*+}$ (fig. $6 \mathrm{~b}$ ). To measure the $\mathrm{K}^{*^{-}}(890)$ production rate we simulate

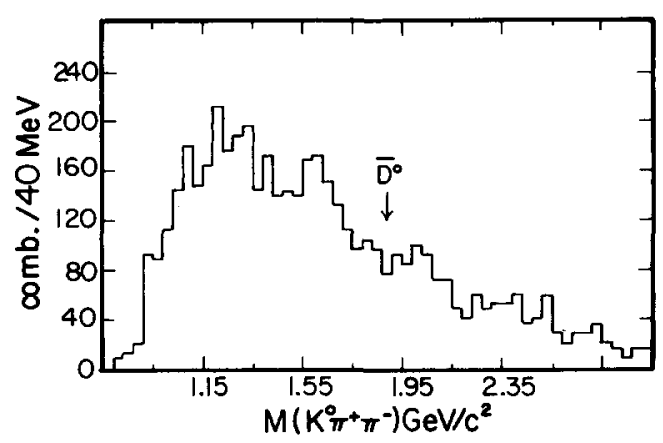

Fig. 7. The $\mathrm{K}^{0} \pi^{+} \pi^{-}$invariant-mass distribution. 
non-resonant $\mathrm{K}^{0} \pi^{-}$background by randomly associating $\mathrm{K}^{0}$ mesons with negative pions from different events which were required to have approximately the same hadronic energy as the $K^{0}$ event. The resulting background distribution was normalized to the $\mathrm{K}^{0} \boldsymbol{\pi}^{-}$mass spectrum in the mass region outside the $\mathrm{K}^{*}$-mass (solid curve in fig. 6). The following inclusive production rate for $\mathrm{K}^{*-}(890) \rightarrow \mathrm{K}^{0} \pi^{-}$in CC $\bar{\nu} \mathrm{N}$ interactions was obtained:

$$
\frac{\bar{\nu} \mathrm{N} \rightarrow \mu^{+} \mathrm{K}^{*-}(890)+\mathrm{X}, \mathrm{K}^{*-} \rightarrow \mathrm{K}^{0} \pi^{-}}{\bar{\nu} \mathrm{N} \rightarrow \mu^{+}+\mathrm{X}}=(0.81 \pm 0.34) \% .
$$

This value is close to the $\mathrm{K}^{*+}(890)$ production rate obtained in neutrino interactions [22].

The effective-mass distributions for the combination $A \pi^{-}$and $\Lambda \pi^{+}$are shown in figs. 8a, b, respectively. We observe a strong signal from the $\Sigma^{-}(1385)$, but there is no indication of the $\Sigma^{+}(1385)$ in the $A \pi^{+}$mass spectrum. This behaviour is also observed in $\bar{\nu}$ interactions [7] and the opposite is seen in $\nu \mathrm{p}$ reactions [11]. By using the same procedure for determination of non-resonant background we obtained the inclusive $\Sigma^{-}(1385) \rightarrow \Lambda \pi^{-}$yield in $\bar{\nu} \mathrm{N} \mathrm{CC}$ interactions of

$$
\frac{\bar{\nu} \mathrm{N} \rightarrow \mu^{+} \Sigma^{-}(1385)+\mathrm{X}, \Sigma^{-} \rightarrow \Lambda \pi^{-}}{\bar{\nu} \mathrm{N} \rightarrow \mu^{+}+\mathrm{X}}=(0.30 \pm 0.19) \%,
$$

which is again in a good agreement with the $\Sigma^{+}(1385)$ production rate obtained in neutrino reactions [22].
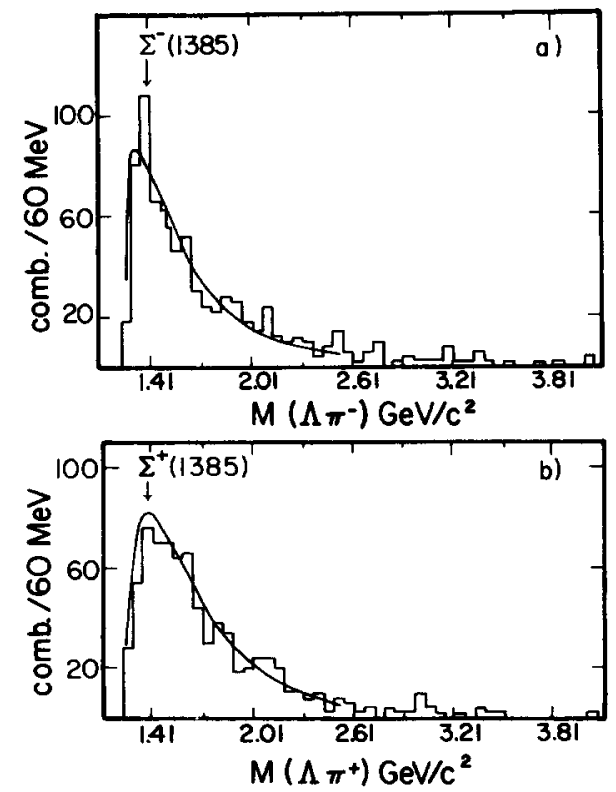

Fig. 8. The effective-mass spectrum for (a) $A \pi^{-}$, (b) $\Lambda \pi^{+}$. The solid curve represents the estimated background. 
Taking into account our data on the $\mathrm{K}^{0}$ and $\Lambda$ inclusive production rates we obtain that only $(5 \pm 2) \%$ of the $\mathrm{K}^{0}$ mesons and $\Lambda$ hyperons result from the decays of $\mathrm{K}^{*^{-}}(890)$ and $\Sigma^{-}(1385)$ resonances, respectively.

\section{5. $\mathrm{K}^{0}$ AND $A$ PRODUCTION ON PROTON AND NEUTRON TARGETS}

The study of neutral strange particle production in antineutrino interactions on proton and neutron targets separately can add to our understanding of the diquark fragmentation processes and the nucleon structure. Measurements of the $\Lambda$ production are particularly important because $\Lambda$ hyperons are the only baryons which can be identified with a high efficiency. Moreover, almost all $\Lambda$ 's are produced in the target fragmentation region [8].

Separation of the $\mathrm{V}^{0}$ production off a proton and neutron target is based on the total final-state charge. The details of this separation procedure have been described elsewhere [23]. This method gives the following results:

$$
\begin{aligned}
& \left\langle n_{\mathrm{K}}^{\mathrm{p}}\right\rangle=0.18 \pm 0.02, \quad\left\langle n_{\mathrm{K}}^{\mathrm{n}}\right\rangle=0.11 \pm 0.02, \\
& \left\langle n_{\Lambda}^{\mathrm{p}}\right\rangle=0.07 \pm 0.01, \quad\left\langle n_{\Lambda}^{\mathrm{n}}\right\rangle=0.05 \pm 0.01,
\end{aligned}
$$

where $\left\langle n_{\mathrm{K}(\Lambda)}^{\mathrm{p}(\mathrm{n})}\right\rangle$ is the average multiplicity of $\mathrm{K}^{0}$ mesons ( $\Lambda$ hyperons) produced in $\bar{\nu} \mathrm{p}$ $(\bar{\nu} \mathrm{n})$ charged current interactions. The obtained results of $\mathrm{K}^{0}$ and $\Lambda$ production on the proton target are in a good agreement with the similar data obtained in $\bar{\nu} \mathrm{p}$ interactions (see table 2).

In fig. 9 we present the $x_{\mathrm{F}}$ dependence of the ratio $R_{\mathrm{p} / \mathrm{n}}^{\mathrm{V}}$ for the $\mathrm{K}^{0} \mathrm{~s}$ (fig. $9 \mathrm{a}$ ) and $\Lambda$ 's (fig. 9b), where $x_{\mathrm{F}}$ is the Feynman scaling variable $x_{\mathrm{F}}=2 p_{\mathrm{L}}^{*} / W$ and $R_{\mathrm{p} / \mathrm{n}}^{\mathrm{V}}=$
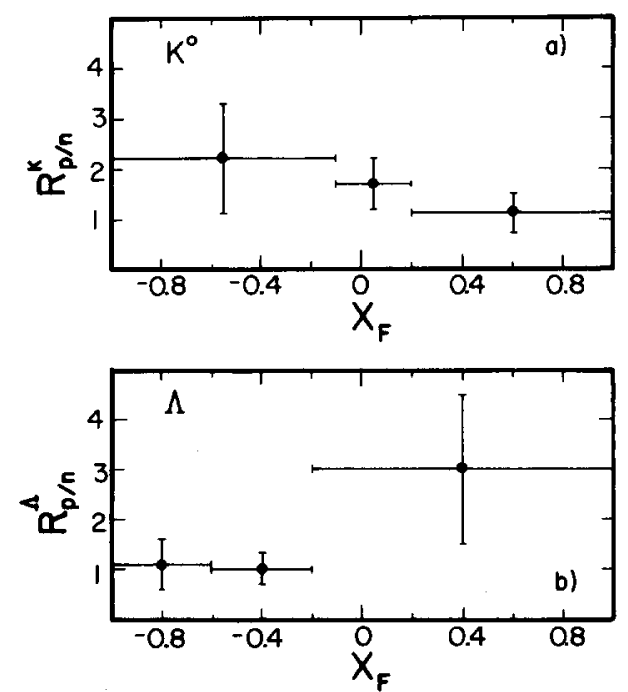

Fig. 9. Relative production rates of (a) $\mathrm{K}^{0 \text { 's }}$ and (b) $\Lambda$ 's from CC interactions off proton and neutron targets as a function of $x_{F}$. 
TABLE 3

Neutral strange particle production on proton and neutron targets, $W^{2}>10 \mathrm{GeV}^{2}$

\begin{tabular}{ccc}
\hline$R_{\mathrm{p} / \mathrm{n}}^{\mathrm{V}}=\left\langle n_{\mathrm{V}}^{\mathrm{P}}\right\rangle /\left\langle n_{\mathrm{V}}^{\mathrm{n}}\right\rangle$ & $x_{\mathrm{F}}<0$ & $x_{\mathrm{F}}>0$ \\
\hline$R_{\mathrm{p} / \mathrm{n}}^{\mathrm{K}}$ & $1.26 \pm 0.49$ & $1.49 \pm 0.37$ \\
$R_{\mathrm{p} / \mathrm{n}}^{\Lambda}$ & $1.16 \pm 0.32$ & \\
\hline
\end{tabular}

Our statistics do not allow us to give a result for the $\Lambda$ 's in the region $x_{\mathrm{F}}>0$.

$\left\langle n_{\mathrm{V}}^{\mathrm{P}}\right\rangle /\left\langle n_{\mathrm{V}}^{\mathrm{n}}\right\rangle$. Here $p_{\mathrm{L}}^{*}$ is the hadron momentum along the current direction in the hadron c.m.s. There is no significant dependence of this ratio versus $x_{F}$ for $K^{0}$ or $\Lambda$ data. For the whole $x_{F}$ interval we have the following results:

$$
R_{\mathrm{p} / \mathrm{n}}^{\mathrm{K}}=1.60 \pm 0.32, \quad R_{\mathrm{p} / \mathrm{n}}^{\Lambda}=1.51 \pm 0.33 .
$$

To remove possible quasi-elastic particle production we have made the selection $W^{2}>10 \mathrm{GeV}^{2}$. The results for the forward and backward c.m.s. hemispheres are shown in table 3 . Within errors, the $\mathrm{K}^{0}$ multiplicity is the same for the proton and neutron targets and for the forward and backward directions.

The experimental result for the $A$ 's in the target fragmentation region $\left(x_{\mathrm{F}}<0\right)$ is unexpected in the naive QPM because in $\bar{\nu}$ CC interactions the speetator diquark system consists of a (ud) pair and in $\bar{\nu}$ CC interactions of a (dd) pair. Therefore, it should be easier to produce a $\Lambda$ hyperon in $\bar{\nu}$ interactions off protons than off neutrons. Our result $R_{\mathrm{p} / \mathrm{n}}^{\Lambda}=1.16 \pm 0.32$ for backward $\Lambda$ 's does not agree with these qualitative expectations.

\section{Summary}

We have performed a detailed study of neutral strange particle production in charged current $\bar{\nu} \mathrm{N}$ interactions. The major results can be summarized as follows:

(1) The overall yield of the CC events with neutral strange particles is $0.16 \pm 0.01$. The inclusive $\mathrm{K}^{0}$ and $A$ production rates are $0.164 \pm 0.009$ and $0.063 \pm 0.004$, respectively. The single $\Lambda$ production is estimated to be $(1.1 \pm 0.7) \%$.

(2) The $W^{2}$ dependence of average $\mathrm{K}^{0}$ and $\Lambda$ multiplicity is well described by a logarithmic form $\left(a+b \ln W^{2}\right)$ or a power-law relation $\left(a+b W^{1 / 2}\right)$. We see no significant $Q^{2}$ dependence of $\left\langle n_{\mathrm{V}}\right\rangle$ in the regions $W^{2}<20 \mathrm{GeV}^{2}$ and $W^{2}>20 \mathrm{GeV}^{2}$.

(3) The average $K^{0}$ multiplicity does not depend on $W^{2}$ or $Q^{2}$ in the current fragmentation region $(z>0.3)$.

(4) In the QPM framework our $\mathrm{V}^{0}$ data are consistent with the charm hypothesis. Fitting $\left\langle n_{\mathrm{V}}\right\rangle$ as functions of $x$ and $y$, the $\mathrm{K}^{0}$ and $A$ multiplicity from the charmed particle decays is found to be $(6 \pm 2) \%$. This result is consistent with dilepton data obtained in this experiment. 
(5) The $\mathrm{K}^{0}$ data in the high- $z$ region $(z>0.3)$ are in agreement with the QPM predictions.

(6) The upper limits for $\mathrm{D}^{-} \rightarrow \mathrm{K}^{0} \pi^{-}$and $\overline{\mathrm{D}}^{0} \rightarrow \mathrm{K}^{0} \pi^{+} \pi^{-}$production rates are measured to be $0.2 \%$ and $0.3 \%$, respectively.

(7) There are signals from $\mathrm{K}^{*^{-}}(890)$ and $\Sigma^{-}(1385)$ resonances in the effectivemass spectra. The observed relative yields are $(0.81 \pm 0.34) \%$ for $\mathrm{K}^{*^{-}} \rightarrow \mathrm{K}^{0} \pi^{-}$and $(0.30 \pm 0.19) \%$ for $\Sigma^{-} \rightarrow \Lambda \pi^{-}$which are in agreement with the $\mathrm{K}^{*+}$ and $\Sigma^{+}$rates obtained in neutrino interactions.

(8) The ratios between $\mathrm{K}^{0}$, s produced off proton and neutron targets in the $W^{2}>10 \mathrm{GeV}^{2}$ region are $1.26 \pm 0.49\left(x_{\mathrm{F}}<0\right)$ and $1.49 \pm 0.37\left(x_{\mathrm{F}}>0\right)$. The value of this ratio for backward $\Lambda$ 's is $1.16 \pm 0.32$, and does not agree with expectations derived from the naive QPM.

We wish to thank the members of the Neutrino Laboratory at Fermilab and the scanning, measuring and secretarial staffs at our respective laboratories for their contribution to this experiment. This work was supported in part by the US Department of Energy and the National Science Foundation.

\section{References}

[1] S.L. Glashow, J. Iliopoulos and L. Maiani, Phys. Rev. D2 (1970) 1285

[2] G.F. Feldman et al., Phys. Rev. Lett. 38 (1977) 1313;

R.W. Brandelik et al., Phys. Lett. 70B (1977) 132;

M. Piccolo et al., Phys. Lett. 70B (1977) 260;

G. Goldhaber et al., Phys. Rev. Lett. 37 (1976) 255;

I. Peruzzi et al., Phys. Rev. Lett. 37 (1976) 569

[3] B. Knapp et al., Phys. Rev. Lett. 37 (1976) 882;

M.S. Atiya et al., Phys. Rev. Lett. 43 (1979) 414

[4] C. Baltay et al., Phys. Rev. Lett. 41 (1978) 73;

C. Baltay et al., Phys. Rev. Lett. 42 (1979) 1721

[5] J.P. Berge et al., Phys. Lett. 81B (1979) 89

[6] R.P. Feynman, Photon-hadron interactions (Benjamin, Reading, Massachusetts, 1972)

[7] S.J. Barish et al., Strange particle production in $\bar{\nu}$ p interactions, Proc. Int. Conf. Neutrino 79, Bergen, 1979, vol. 2, p. 582

[8] V.V. Ammosov et al., Nucl. Phys. B162 (1980) 205

[9] D. Sinclair, Measurement of $x$ and $y$ distributions for antineutrino charged current inelastic scattering, Proc. Int. Conf. Neutrino 79, Bergen, 1979 vol. 2, p. 343

[10] R.B. Palmer, Lepton and charm production in the $15 \mathrm{ft}$ Bubble Chamber, Proc. 13th Recontre de Moriond, Les Arcs-Savoie, 1978 (28100 Dreux, France, 1978) vol. 2, p. 361

[11] J.P. Berge et al., Phys. Rev. D18 (1978) 1359

[12] M. Derrick et al., Phys. Rev. D17 (1978) 17

[13] R. Orava, ABCMO $\nu$ p data, private communication

[14] L. Pape, Estimation of charm production and lifetime limits in $\nu$ interactions at BEBC, Proc. Topical Conf. on Neutrino physics at accelerators, Oxford, 1978 (Pub. RL-78-081) 167

[15] B. Cork and T.F. Hoang, Multiplicity and Scaling of $\mathrm{e}^{+} \mathrm{e}^{-} \rightarrow$ hadrons, preprint LBL-9958 (1979)

[16] R.D. Field and R.P. Feynman, Phys. Rev. D15 (1977) 2590; Nucl. Phys. B136 (1978) 1

[17] F. Messing, Strange particle production in lepton-proton interactions, Proc. Topical Conf. on Neutrino physics at accelerators, Oxford, 1978 (Pub. RL-78-081) 26 
[18] I. Cohen et al., Phys. Rev. Lett. 40 (1978) 1614

[19] C.-H. Lai, Phys. Rev. D18 (1978) 1422

[20] Review of Particle Properties, Phys. Lett. 75B (1978) 1

[21] C. Quigg and J.L. Rosner, Phys. Rev. D17 (1978) 239

[22] P. Schreiner, Particle production by neutrinos, Proc. Int. Symp. on Lepton and photon interactions at high energies, Fermilab, 1979, p. 291

[23] J.P. Berge et al., Phys. Lett. 84B (1979) 511 